\title{
An Extension of the Mittag-Leffler Function and Its Associated Properties
}

\author{
Gauhar Rahman, ${ }^{1}$ Abdus Saboor, ${ }^{2}$ Zunaira Anjum, ${ }^{2}$ Kottakkaran Sooppy Nisar, ${ }^{3}$ \\ and Thabet Abdeljawad (iD) $4,5,6$
}

${ }^{1}$ Department of Mathematics, Shaheed Benazir Bhutto University, Sheringal 18000, Upper Dir, Pakistan
${ }^{2}$ Institute of Numerical Sciences, Kohat University of Science \& Technology, Kohat, Pakistan 26000
${ }^{3}$ Department of Mathematics, College of Arts and Sciences, Prince Sattam Bin Abdulaziz University,
Wadi Aldawser 11991, Saudi Arabia
${ }^{4}$ Department of Mathematics and General Sciences, Prince Sultan University, Riyadh, Saudi Arabia
${ }^{5}$ Department of Medical Research, China Medical University, Taichung 40402, Taiwan
${ }^{6}$ Department of Computer Science and Information Engineering, Asia University, Taichung 40402, Taiwan

Correspondence should be addressed to Thabet Abdeljawad; tabdeljawad@psu.edu.sa

Received 27 June 2020; Revised 17 August 2020; Accepted 29 August 2020; Published 22 September 2020

Academic Editor: Soheil Salahshour

Copyright (c) 2020 Gauhar Rahman et al. This is an open access article distributed under the Creative Commons Attribution License, which permits unrestricted use, distribution, and reproduction in any medium, provided the original work is properly cited.

Inspired by certain fascinating ongoing extensions of the special functions such as an extension of the Pochhammer symbol and generalized hypergeometric function, we present a new extension of the generalized Mittag-Leffler (ML) function $\varepsilon_{a, b ; p, v}^{\kappa}\left(z_{1}\right)$ in terms of the generalized Pochhammer symbol. We then deliberately find certain various properties and integral transformations of the said function $\varepsilon_{a, b ; p, v}^{\kappa}\left(z_{1}\right)$. Some particular cases and outcomes of the main results are also established.

\section{Introduction}

The well-known ML (Mittag-Leffler) function with one parameter is defined by

$$
\varepsilon_{a}\left(z_{1}\right)=\sum_{l=0}^{\infty} \frac{z_{1}^{l}}{\Gamma(a l+1)}\left(a \in \mathbb{C} ; \mathfrak{R}(a)>0, z_{1} \in \mathbb{C}\right) .
$$

The generalization of (1) with two parameters defined by

$$
\varepsilon_{a, b}\left(z_{1}\right)=\sum_{l=0}^{\infty} \frac{z_{1}^{l}}{\Gamma(a l+b)}(a, b \in \mathbb{C} ; \mathfrak{R}(a)>0, \mathfrak{R}(b)>0)
$$

was presented and contemplated by Mittag-Leffler [1-7] and other researchers. In [8], the generalization of (1) was given by

$$
\varepsilon_{a, b}^{\kappa}\left(z_{1}\right)=\sum_{l=0}^{\infty} \frac{(\kappa)_{l}}{\Gamma(a l+b)} \frac{z_{1}^{l}}{l !}(a, b, \kappa \in \mathbb{C} ; \mathfrak{R}(a)>0, \mathfrak{R}(b)>0) .
$$

Shukla and Prajapati [9] defined the following generalization of the ML function by

$$
\varepsilon_{a, b}^{\kappa, q}\left(z_{1}\right)=\sum_{l=0}^{\infty} \frac{(\kappa)_{l q}}{\Gamma(a l+b)} \frac{z_{1}^{l}}{l !}(a, b, \kappa \in \mathbb{C} ; \mathfrak{R}(a)>0, \mathfrak{R}(b)>0, q>0) .
$$

Rahman et al. [10] defined the following extension of generalized ML function by

$$
\varepsilon_{a, b, c}^{\kappa, q, c}\left(z_{1}\right)=\sum_{l=0}^{\infty} \frac{B(\kappa+l q ; c-\kappa)(c)_{l q}}{B(\kappa, c-\kappa) \Gamma(a l+b)} \frac{z_{1}^{l}}{l !}
$$

where $a, b, \kappa, c \in \mathbb{C} ; \mathfrak{R}(c)>0, \mathfrak{R}(a)>0, \mathfrak{R}(b)>0, \mathfrak{R}(p)>0$, 
$q>0$, and $B_{p}(x, y)$ is the extension of beta function (see [11]).

Moreover, the generalization of ML function (3) was presented by [12] as follows:

$\varepsilon_{a, b, p}^{\kappa}\left(z_{1}\right)=\sum_{l=0}^{\infty} \frac{(\kappa ; p)_{l}}{\Gamma(a l+b)} \frac{z_{1}^{l}}{l !}(p \geq 0, a, b, \kappa \in \mathbb{C} ; \Re(a)>0, \mathfrak{R}(b)>0, \mathfrak{R}(p)>0)$,

where $(\kappa ; p)_{l}$ is the Pochhammer symbol which is defined as

$$
(\lambda ; \sigma)_{\mu}= \begin{cases}\frac{\Gamma_{\sigma}(\lambda+\mu)}{\Gamma(\lambda)}, & \mathfrak{R}(\sigma), \lambda, \mu \in \mathbb{C} \\ (\lambda)_{\mu}, & \sigma=0, \lambda, \mu \in \mathbb{C} \backslash\{0\} .\end{cases}
$$

The researchers studied these extensions (6) and (7) and investigated their further extensions and associated properties and applications. (The readers may consult [13-16].) Recently, Srivastava et al. [17] have presented and concentrated in a fairly productive way the following extension of the generalized hypergeometric function:

$$
\begin{aligned}
{ }_{s} F_{t} & {\left[\left(\delta_{1} ; \rho, v\right), \cdots,\left(\delta_{s}\right) ;\left(\zeta_{1}\right), \cdots,\left(\zeta_{t}\right) ; z_{1}\right] } \\
= & \sum_{l=0}^{\infty} \frac{\left(\delta_{1} ; \rho, v\right)_{l} \cdots\left(\delta_{s}\right)_{l}}{\left(\zeta_{1}\right)_{l} \cdots\left(\zeta_{t}\right)_{l}} \frac{z_{1}^{l}}{l !}
\end{aligned}
$$

where $\delta_{j} \in \mathbb{C}$ for $j=1,2, \cdots, s, \zeta_{k} \in \mathbb{C}$ for $k=1,2, \cdots, t$, and $\zeta_{k} \neq 0,-1,-2, \cdots$, and where $(\mu ; \omega, a)_{\eta}$ is the extension of the generalized Pochhammer symbol defined by [23]:

$$
(\mu ; \omega, a)_{\eta}= \begin{cases}\frac{\Gamma_{a}(\mu+\eta ; \omega)}{\Gamma(\mu)}, & \Re(\omega), \mathfrak{R}(a)>0, \mu, \eta \in \mathbb{C}, \\ (\mu ; \omega)_{\eta}, & a=0, \mu, \eta \in \mathbb{C} \backslash\{0\} .\end{cases}
$$

The integral representation of $(\mu ; \omega, a)_{\eta}$ is explained by

$$
(\mu ; \omega, a)_{\eta}=\sqrt{\frac{2 \omega}{\pi}} \frac{1}{\Gamma(\mu)} \int_{0}^{\infty} s^{\mu+\eta-3 / 2} e^{-s} K_{a+1 / 2}\left(\frac{\omega}{s}\right) d s
$$

where $K_{a}(\cdot)$ is the modified Bessel function of order $a$. Clearly, when $a=0$ in (10), at that point, by utilizing the way that $K_{1 / 2}(t)=\sqrt{\pi / 2 t} e^{-t}$, it will lead to formula [(31)]:

$$
(\mu ; \omega, 0)_{\eta}=(\mu ; \omega)_{\eta}=\frac{1}{\Gamma(\mu)} \int_{0}^{\infty} s^{\mu+\eta-1} e^{-s} d s .
$$

Specifically, the relating extensions of the confluent hypergeometric function $1 F_{1}$ and the Gauss hypergeometric function ${ }_{2} F_{1}$ are given by

$$
{ }_{2} F_{1}\left[\left(\delta_{1} ; \rho, v\right), b ; \kappa ; z_{1}\right]=\sum_{l=0}^{\infty} \frac{\left(\delta_{1} ; \rho, v\right)_{l}(b)}{(\kappa)_{l}} \frac{z_{1}^{l}}{l !}
$$

${ }_{1} F_{1}\left[\left(\delta_{1} ; \rho, v\right) ; \kappa ; z_{1}\right]=\Phi\left[\left(\delta_{1} ; \rho, v\right) ; \kappa ; z_{1}\right]=\sum_{l=0}^{\infty} \frac{\left(\delta_{1} ; \rho, v\right)_{l}}{(\kappa)_{l}} \frac{z_{1}^{l}}{l !}$

The extension of generalized hypergeometric function ${ }_{r} F_{s}$ of $r$ numerator and $s$ denominator parameters was investigated by [18]. Recently, the researchers defined various extensions of special functions and their associated properties and applications in the diverse field. (The interested readers may consult [19-22].) In [23-25], the authors introduced an extension of fractional derivative operators based on the extended beta functions.

Next, motivated by the above such extensions of special functions, we define an extension of ML function (6) in terms of the generalized Pochhammer symbol (9) and investigate its certain variations.

\section{Extension of ML Function}

We present an extension of the generalized ML function in (6) regarding the extended Pochhammer symbol in (9) as follows:

$$
\begin{aligned}
\varepsilon_{a, b ; p, v}^{\kappa}\left(z_{1}\right)= & \sum_{l=0}^{\infty} \frac{(\kappa ; p, v)_{l}}{\Gamma(a l+b)} \frac{z_{1}^{l}}{l !}(a, b, \kappa \in \mathbb{C} ; \mathfrak{R}(a)>0, \mathfrak{R}(b)>0, \\
& \Re(p)>0, p \geq 0, v \geq 0),
\end{aligned}
$$

given that the series on the right hand side converges.

Clearly, it diminishes to the extended generalized $\mathrm{ML}$ function (6) for $v=0$. The special case for $a=1$ in (14) can be communicated regarding extended confluent hypergeometric function (13) as follows:

$$
\begin{aligned}
\varepsilon_{1, b ; p, v}^{\kappa}\left(z_{1}\right) & =\frac{1}{\Gamma(b)_{1}} F_{1}\left[\left(\kappa_{1} ; p, v\right) ; b ; z_{1}\right] \\
& =\frac{1}{\Gamma(b)} \Phi\left[\left(\kappa_{1} ; p, v\right) ; b ; z_{1}\right] .
\end{aligned}
$$

\section{Basic Properties of $\varepsilon^{\kappa}{ }_{a, b ; p, v}\left(z_{1}\right)$}

In this section, we present certain basic properties and integral representations of the extended generalized ML function $\varepsilon_{a, b ; p, v}^{\kappa}\left(z_{1}\right)$ in (14).

Theorem 1. For the function $\varepsilon_{a, b ; p, v}^{\kappa}\left(z_{1}\right)$ in (14), the following relation holds true:

$$
\begin{gathered}
\varepsilon_{a, b ; p, v}^{\kappa}\left(z_{1}\right)=b \varepsilon_{a, b+1 ; p, v}^{\kappa}\left(z_{1}\right)+a z_{1} \frac{d}{d z} \varepsilon_{a, b+1 ; p, v}^{\kappa}\left(z_{1}\right) \\
\cdot(a, b, \kappa \in \mathbb{C} ; \mathfrak{R}(a)>0, \mathfrak{R}(b)>0, \\
\mathfrak{R}(p)>0, p \geq 0, v \geq 0) .
\end{gathered}
$$


Specifically, we have

$$
E_{a, b ; p}^{\kappa}\left(z_{1}\right)=b E_{a, b+1 ; p}^{\kappa}\left(z_{1}\right)+a z_{1} \frac{d}{d z_{1}} E_{a, b+1 ; p}^{\kappa}\left(z_{1}\right) .
$$

Proof. From (14), we have

$$
\begin{aligned}
b \varepsilon_{a, b+1, p, v}^{\kappa}( & \left.z_{1}\right)+a z_{1} \frac{d}{d z_{1}} \varepsilon_{a, b+1, p, v}^{\kappa}\left(z_{1}\right) \\
& =b \sum_{l=0}^{\infty} \frac{(\kappa, p, v)_{l}}{\Gamma(a l+b+1)} \frac{z_{1}^{l}}{l !}+a z_{1} \frac{d}{d z_{1}} \sum_{l=0}^{\infty} \frac{(\kappa, p, v)_{l}}{\Gamma(a l+b+1)} \frac{z_{1}^{l}}{l !} \\
& =b \sum_{l=0}^{\infty} \frac{(\kappa, p, v)_{l}}{\Gamma(a l+b+1)} \frac{z_{1}^{l}}{l !}+a z_{1} \frac{d}{d z_{1}} \sum_{l=0}^{\infty} \frac{(\kappa, p, v)_{l}}{\Gamma(a l+b+1)} \frac{z_{1}^{l}}{l !} \\
& =b \sum_{l=0}^{\infty} \frac{(\kappa, p, v)_{l}}{\Gamma(a l+b+1)} \frac{z_{1}^{l}}{l !}+\sum_{l=0}^{\infty} \frac{a z_{1}(\kappa, p, v)_{l} l}{\Gamma(a l+b+1)} \frac{z^{l-1}}{l !} \\
& =b \sum_{l=0}^{\infty} \frac{(\kappa, p, v)_{l}}{\Gamma(a l+b+1)} \frac{z_{1}^{l}}{l !}+\sum_{l=0}^{\infty} \frac{(\kappa, p, v)_{l}(a l)}{\Gamma(a l+b+1)} \frac{z_{1}^{l}}{l !} \\
& =\sum_{l=0}^{\infty} \frac{(\kappa, p, v)_{l}}{\Gamma(a l+b+1)} \frac{z_{1}^{l}}{l !}(a l+b) \\
& =\sum_{l=0}^{\infty} \frac{(\kappa, p, v)_{l}(a l+b)}{(a l+b) \Gamma(a l+b)} \frac{z_{1}^{l}}{l !}, \text { using } \Gamma\left(z_{1}+1\right) \\
& =z_{1} \Gamma\left(z_{1}\right),=\sum_{l=0}^{\infty} \frac{(\kappa, p, v)_{l}}{\Gamma(a l+b)} \frac{z_{1}^{l}}{l !}=\varepsilon_{a, b, p, v}^{\kappa}\left(z_{1}\right) .
\end{aligned}
$$
$v=0$.

Equation (17) can be obtained from (16) when we put

Theorem 2. For the function $\varepsilon_{a, b ; p, v}^{\kappa}\left(z_{1}\right)$ in (14), the following higher order differentiation formulas hold true:

$$
\begin{gathered}
\left(\frac{d}{d z_{1}}\right)^{m} \varepsilon_{a, b ; p, v}^{\kappa}\left(z_{1}\right)=(\kappa)_{m} \varepsilon_{a, b+m ; p, v}^{\kappa+m}\left(z_{1}\right), \\
\left(\frac{d}{d z_{1}}\right)^{m}\left[z_{1}^{b-1} \varepsilon_{a, b ; p, v}^{\kappa}\left(\omega z_{1}^{a}\right)\right] \\
=z_{1}^{b-m-1} \varepsilon_{a, b-m ; p, v}^{\kappa}\left(\omega z_{1}^{a}\right)(\Re(b-m)>0, m \in \mathbb{N}), \\
\left(\frac{d}{d z_{1}}\right)^{m}\left[z_{1}^{b-1} \Phi\left((\kappa ; p, v) ; b ; \omega z_{1}\right)\right] \\
=\frac{\Gamma(b)}{\Gamma(b-m)} z_{1}^{b-m-1} \Phi\left((\kappa ; p, v) ; b-m ; \omega z_{1}\right)
\end{gathered}
$$

where $a, b, \kappa \in \mathbb{C} ; \mathfrak{R}(a)>0, \mathfrak{R}(b)>0, \mathfrak{R}(p)>0, p \geq 0, v \geq 0$.

Specifically, we have

$$
\begin{aligned}
\left(\frac{d}{d z_{1}}\right)^{m} E_{a, b, p}^{\kappa}\left(z_{1}\right) & =(\kappa)_{m} E_{a, b+m, p}^{\kappa+m}\left(z_{1}\right), \\
\left(\frac{d}{d z_{1}}\right)^{m}\left[z_{1}^{b-1} E_{a, b, p}^{\kappa}\left(\omega z_{1}^{a}\right)\right] & =z_{1}^{b-m-1} E_{a, b-m, p}^{\kappa}\left(\omega z_{1}^{a}\right),
\end{aligned}
$$

$$
\begin{aligned}
\left(\frac{d}{d z_{1}}\right)^{m} & {\left[z_{1}^{b-1} \Phi\left((\kappa, p) ; b ; \omega z_{1}\right)\right] } \\
& =\frac{\Gamma(b)}{\Gamma(b-m)} z_{1}^{b-m-1} \Phi\left((\kappa, p) ; b-m ; \omega z_{1}\right) .
\end{aligned}
$$

Proof. Operating term wise differentiation $m$ times on (14), we get

$$
\begin{aligned}
\left(\frac{d}{d z_{1}}\right)^{m} \varepsilon_{a, b ; p, v}^{\kappa}\left(z_{1}\right) & =\left(\frac{d}{d z_{1}}\right)^{m} \sum_{l=0}^{\infty} \frac{(\kappa ; p, v)_{l}}{\Gamma(a l+b)} \frac{z_{1}^{l}}{l !} \\
& =\sum_{l=m}^{\infty} \frac{(\kappa ; p, v)_{l}}{\Gamma(a l+b)} \frac{l ! z^{l-m}}{(l-m) ! l !} \\
& =\sum_{l=0}^{\infty} \frac{(\kappa ; p, v)_{l+m}}{\Gamma(a l+b+a m)} \frac{z_{1}^{l}}{(l) !}, \text { replacing } l \text { by } l+m, \\
& =\sum_{l=0}^{\infty} \frac{(\kappa)_{m}(\kappa+m ; p, v)_{l}}{\Gamma(a l+b+a m)} \frac{z_{1}^{l}}{l !} \text { using }(\lambda ; \sigma, v)_{\mu+p} \\
& =(\lambda)_{\mu}(\lambda+\mu ; \sigma, v)_{p}, \\
& =(\kappa)_{m} \sum_{l=0}^{\infty} \frac{(\kappa+m ; p, v)_{n}}{\Gamma(a l+b+a m)} \frac{z_{1}^{l}}{l !} \\
& =(\kappa)_{m} \varepsilon_{a, b+m ; p, v}^{\kappa+m}\left(z_{1}\right) .
\end{aligned}
$$

In a similar manner from (20), we get

$$
\begin{aligned}
\left(\frac{d}{d z_{1}}\right)^{m} & {\left[z^{b-1} \varepsilon_{a, b ; p, v}^{\kappa}\left(\omega z_{1}^{a}\right)\right] } \\
& =\left(\frac{d}{d z_{1}}\right)^{m} z_{1}^{b-1} \sum_{l=0}^{\infty} \frac{(\kappa ; p, v)_{l}}{\Gamma(a l+b)} \frac{\left(\omega z_{1}^{a}\right)^{l}}{l !} \\
& =\left(\frac{d}{d z_{1}}\right)^{m} \sum_{l=0}^{\infty} \frac{(\kappa ; p, v)_{l} z_{1}^{b-1+a l}}{\Gamma(a l+b)} \omega^{l} \\
& =\sum_{l=0}^{\infty} \frac{(\kappa ; p, v)_{l}}{\Gamma(a l+b)} \frac{\omega^{l}}{l !} \frac{(a l+b-1) !}{(a l+b-m-1) !} z_{1}^{a l+b-m-1}, \\
& =\sum_{l=0}^{\infty} \frac{(\kappa ; p, v)_{l}}{\Gamma(a l+b)} \frac{\Gamma(a l+b)}{\Gamma(a l+b-m)} \omega^{l} \frac{z_{1}^{a l+b-1-m}}{l !}, \\
& =z_{1}^{b-m-1} \sum_{l=0}^{\infty} \frac{(\kappa ; p, v)_{l}}{\Gamma(a l+b-m)} \frac{\left(\omega z_{1}^{a}\right)^{l}}{l !} \\
& =z_{1}^{b-m-1} \varepsilon_{a, b-m ; p, v}^{\kappa}\left(\omega z_{1}^{a}\right) .
\end{aligned}
$$

Moreover, putting $a=1$ in (20) gives (21). For the special case of (19), (20), and (21), when we put $v=0$, we get (22), (23), and (24), respectively.

Corollary 3. The following integral representations for $M L$ function $\varepsilon_{a, b ; p, v}^{\kappa}\left(z_{1}\right)$ (14) hold true: 


$$
\begin{gathered}
\int_{0}^{z_{1}} t^{b-1} \varepsilon_{a, b ; p, v}^{\kappa}\left(\omega t^{a}\right) d t=z_{1}^{b} \varepsilon_{a, b+1 ; p, v}^{\kappa}\left(\omega z_{1}^{a}\right), \\
\int_{0}^{z_{1}} t^{b-1} \Phi((\kappa, p, v) ; b ; \omega t) d t=\frac{1}{\Gamma(b)} z^{b} \Phi\left((\kappa ; p, v) ; b+1 ; \omega z_{1}\right),
\end{gathered}
$$

where $a, b, \kappa, \omega \in \mathbb{C} ; \mathfrak{R}(a)>0, \mathfrak{R}(b)>0, \mathfrak{R}(p)>0, p \geq 0, v \geq$ 0 .

Specifically, we have

$$
\begin{aligned}
\int_{0}^{z_{1}} t^{b-1} E_{a, b ; p}^{\kappa}\left(\omega t^{a}\right) d t & =z_{1}^{b} E_{a, b+1 ; p}^{\kappa}\left(\omega z_{1}^{a}\right), \\
\int_{0}^{z_{1}} t^{b-1} \Phi((\kappa, p) ; b ; \omega t) d t & =\frac{1}{\Gamma(b)} z^{b} \Phi\left((\kappa ; p) ; b+1 ; \omega z_{1}\right) .
\end{aligned}
$$

\section{Representation of $\varepsilon_{a, b ; p, v}^{\kappa}\left(z_{1}\right)$ in terms of Generalized Hypergeometric Function}

Here, we establish the representation of $\varepsilon_{a, b ; p, v}^{\kappa}\left(z_{1}\right)(14)$ in terms of generalized hypergeometric function as follows.

Theorem 4. The function $\varepsilon_{a, b ; p, v}^{\kappa}\left(z_{1}\right)$ defined in (14) for $a \in N$ can be represented in the form of generalized hypergeometric function as given by

$$
\begin{aligned}
\varepsilon_{a, b, p, v}^{\kappa}\left(z_{1}\right) & =\frac{1}{\Gamma(b)_{1}} F_{q}\left[(\kappa, p, v) ; \frac{b}{q}, \frac{b+1}{q}, \cdots, \frac{b+k-1}{q} ; \frac{z_{1}}{q^{q}}\right] \\
& =\frac{1}{\Gamma(b)_{1}} F_{q}\left[(\kappa, p, v) ; \Delta(q ; b) ; \frac{z_{1}}{q^{q}}\right],
\end{aligned}
$$

where $q \in \mathbb{N}$ and $\Delta(q ; b)$ is an array ${ }_{p} f_{q}$ parameters $b / q$, $(b+1) / q, \cdots,(b+q-1) / q$.

Proof. Taking $a=q \in \mathbb{N}$ in (14) and utilizing the well-known multiplication formula for the gamma function, we have

$$
\begin{aligned}
\varepsilon_{q, b, p, v}^{\kappa}\left(z_{1}\right) & =\sum_{l=0}^{\infty} \frac{(\kappa, p, v)_{l}}{\Gamma(q n+b)} \frac{z_{1}^{l}}{l !}=\sum_{l=0}^{\infty} \frac{(\kappa, p, v)_{l}}{(\Gamma(b) / \Gamma(b)) \times \Gamma(q n+b)} \frac{z_{1}^{l}}{l !} \\
& =\frac{1}{\Gamma(b)} \sum_{l=0}^{\infty} \frac{(\kappa, p, v)_{l}}{\Gamma(q n+b) / \Gamma(b)} \frac{z_{1}^{l}}{l !}=\frac{1}{\Gamma(b)} \sum_{l=0}^{\infty} \frac{(\kappa, p, v)_{l}}{(b)_{n q}} \frac{z_{1}^{l}}{l !} \\
& =\frac{1}{\Gamma(b)} \sum_{l=0}^{\infty} \frac{(\kappa, p, v)_{l}}{\prod_{j=1}^{q}((b+j-1) / q)_{n}}\left(\frac{z_{1}}{q^{q}}\right)^{l} \frac{1}{l !}, \text { since }(b)_{q n} \\
& =\prod_{j=1}^{q}\left(\frac{b+j-1}{q}\right)_{n}, \\
& =\frac{1}{\Gamma(b)} \sum_{l=0}^{\infty} \frac{(\kappa, p, v)_{l}}{(b / q)_{l},((b+1) / q)_{l}, \cdots,((b+q-1) / q)_{l}}\left(\frac{z_{1}}{q^{q}}\right)^{l} \frac{1}{l !} \\
& =\frac{1}{\Gamma(b)_{1}} F_{q}\left[(\kappa, p, v) ; \frac{b}{q}, \frac{b+1}{q}, \cdots, \frac{b+q-1}{q} ; \frac{z_{1}}{q^{q}}\right] \\
& =\frac{1}{\Gamma(b)_{1}} F_{q}\left[(\kappa, p, v) ; \Delta(q ; b) ; \frac{z_{1}}{q^{q}}\right] .
\end{aligned}
$$

\section{Integral Transformation of $\varepsilon_{a, b, p, v}^{\kappa}\left(z_{1}\right)$}

Here, we present various integral representations of the function $\varepsilon_{a, b ; p, v}^{\kappa}\left(z_{1}\right)$ in (14) such as the Mellin, the Euler-beta, and the Laplace transformations.

5.1. Mellin Transform. The well-known Millen transform [26] of integrable function $f\left(z_{1}\right)$ with index $s$ is defined by

$$
\mathscr{M}\left\{f\left(z_{1}\right): z_{1} \rightarrow s\right\}=\int_{0}^{\infty} z_{1}^{s-1} f\left(z_{1}\right) d z_{1}
$$

if the improper integral in (31) exists.

Theorem 5. For the function $\varepsilon_{a, b ; p, v}^{\kappa}\left(z_{1}\right)$ in (14), the following Mellin transform exists:

$$
\mathscr{M}\left\{\varepsilon_{a, b, p, v}^{\kappa}\left(z_{1}\right): v \rightarrow s\right\}
$$

$\frac{2^{s-1}}{\sqrt{\pi}} \Gamma\left(\frac{s-v}{2}\right) \Gamma\left(\frac{s+v+1}{2}\right)(\kappa)_{s} E_{a, b}^{\kappa+s}\left(z_{1}\right)(\mathfrak{R}(s-v)>0, \mathfrak{R}(\kappa+s)>-1)$.

Proof. By (32), we get from (14)

$$
\begin{aligned}
\mathscr{M}\left\{\varepsilon_{a, b ; p, v}^{\kappa}\left(z_{1}\right): v \rightarrow s\right\} & =\int_{0}^{\infty} v^{s-1} \varepsilon_{a, b ; p, v}^{\kappa}\left(z_{1}\right) d v \\
& =\int_{0}^{\infty} v^{s-1}\left(\sum_{l=0}^{\infty} \frac{(\kappa ; p, v)_{l}}{\Gamma(a l+b)} \frac{z_{1}^{l}}{l !}\right) d v \\
& =\int_{0}^{\infty} v^{s-1}\left(\sum_{l=0}^{\infty} \frac{\Gamma_{v}(\kappa+l ; p)}{\Gamma(\kappa) \Gamma(a l+b)} \frac{z_{1}^{l}}{l !}\right) d v .
\end{aligned}
$$

Interchanging the order of summation and integration, we get

$$
\mathscr{M}\left\{\varepsilon_{a, b ; p, v}^{\kappa}\left(z_{1}\right): v \rightarrow s\right\}=\sum_{l=0}^{\infty} \frac{1}{\Gamma(a l+b)} \frac{z_{1}^{l}}{l !} \frac{1}{\Gamma(\kappa)} \int_{0}^{\infty} v^{s-1} \Gamma_{v}(\kappa+l ; p) d v
$$
4.105),

$$
\begin{aligned}
\int_{0}^{\infty} v^{s-1} \Gamma_{v}(\kappa+l ; p) d v= & \frac{2^{s-1}}{\sqrt{\pi}} \Gamma\left(\frac{s-v}{2}\right) \Gamma\left(\frac{s+v+1}{2}\right) \\
& \cdot \Gamma(\kappa+s+l)(\mathfrak{R}(\kappa+s)>-1, \mathfrak{R}(s-v)>0) .
\end{aligned}
$$


Therefore, we have

$$
\begin{aligned}
\mathscr{M} & \left\{\varepsilon_{a, b ; p, v}^{\kappa}\left(z_{1}\right): v \rightarrow s\right\} \\
& =\frac{\left(2^{s-1} / \sqrt{\pi}\right) \Gamma((s-v) / 2) \Gamma((s+v+1) / 2)}{\Gamma(\kappa)} \sum_{l=0}^{\infty} \frac{\Gamma(\kappa+s+l)}{\Gamma(a l+b)} \frac{z_{1}^{l}}{l !} \\
& =\frac{2^{s-1}}{\sqrt{\pi}} \Gamma\left(\frac{s-v}{2}\right) \Gamma\left(\frac{s+v+1}{2}\right)(\kappa)_{s} \sum_{l=0}^{\infty} \frac{(\kappa+s)_{l}}{\Gamma(a l+b)} \frac{z_{1}^{l}}{l !} \\
& =\frac{2^{s-1}}{\sqrt{\pi}} \Gamma\left(\frac{s-v}{2}\right) \Gamma\left(\frac{s+v+1}{2}\right)(\kappa)_{s} E_{a, b}^{\kappa+s}\left(z_{1}\right) .
\end{aligned}
$$

Corollary 6. The below integral transform exists:

$$
\begin{aligned}
\mathscr{M}\left\{\varepsilon_{a, b ; p, v}^{\kappa}\left(z_{1}\right): v \rightarrow s\right\} \\
=\frac{\left(2^{s-1} / \sqrt{\pi}\right) \Gamma((s-v) / 2) \Gamma((s+v+1) / 2)}{\Gamma(\kappa)} \\
{ }_{1} \Psi_{1}[(\kappa+s, 1) ;(b, a) ; z],
\end{aligned}
$$

where ${ }_{p} \Psi_{q}$ is the Wright hypergeometric function (see [27, 28]).

5.2. Euler-Beta Transform. In [26], the well-known Eulerbeta transform of the function $f\left(z_{1}\right)$ is defined by

$$
\mathscr{B}\left\{f\left(z_{1}\right) ; a_{1}, b_{1}\right\}=\int_{0}^{1} z_{1}^{a_{1}-1}\left(1-z_{1}\right)^{b_{1}-1} f\left(z_{1}\right) d z_{1} .
$$

Theorem 7. For the function $\varepsilon_{a, b ; p, v}^{\kappa}\left(z_{1}\right)$ in (14), the following Euler-beta transform holds:

$$
\begin{aligned}
\mathscr{B}\left\{\varepsilon_{a, b ; p, v}^{\kappa}\left(x z_{1}^{\sigma}\right): a_{1}, b_{1}\right\} \\
=\frac{\Gamma\left(b_{1}\right) \Gamma\left(a_{1}\right)}{\Gamma(b) \Gamma\left(a_{1}+b_{1}\right)_{\sigma+1}} F_{a+\sigma}\left[(\kappa, p, v), \Delta\left(\sigma ; a_{1}\right) ; \Delta(a ; b), \Delta\left(\sigma ; a_{1}+b_{1}\right) ; \frac{x}{a^{a}}\right] \\
\quad \cdot\left(a, \sigma \in \mathbb{N} ; \mathfrak{R}(p)>0, \mathfrak{R}\left(a_{1}\right)>0, \mathfrak{R}\left(b_{1}\right)>0, \mathfrak{R}(b)>0, \mathfrak{R}(\kappa)>0, p \geq 0, v \geq 0\right) .
\end{aligned}
$$

Proof. By using (40) and (14), we obtain

$$
\begin{aligned}
\mathscr{B} & \left\{\varepsilon_{a, b ; p, v}^{\kappa}\left(x z_{1}^{\sigma}\right): a_{1}, b_{1}\right\} \\
& =\int_{0}^{1} z_{1}^{a_{1}-1}\left(1-z_{1}\right)^{b_{1}-1} \varepsilon_{a, b ; p, v}^{\kappa}\left(x z_{1}^{\sigma}\right) d z_{1} \\
& =\int_{0}^{1} z_{1}^{a_{1}-1}\left(1-z_{1}\right)^{b_{1}-1}\left(\sum_{l=0}^{\infty} \frac{(\kappa ; p, v)_{l}}{\Gamma(a l+b)} \frac{x^{l} z_{1}^{\sigma l}}{l !}\right) d z_{1} \\
& =\sum_{l=0}^{\infty} \frac{(\kappa ; p, v)_{l}}{\Gamma(a l+b)} \frac{x^{l}}{l !}\left(\int_{0}^{1} z_{1}^{a_{1}+\sigma l-1}\left(1-z_{1}\right)^{b_{1}-1}\right) d z_{1} \\
& =\sum_{l=0}^{\infty} \frac{(\kappa ; p, v)_{l}}{\Gamma(a l+b)} \frac{x^{l}}{l !} \frac{\Gamma\left(\sigma l+a_{1}\right) \Gamma\left(b_{1}\right)}{\Gamma\left(\sigma l+a_{1}+b_{1}\right)}
\end{aligned}
$$

$$
\begin{aligned}
= & \frac{\Gamma\left(b_{1}\right) \Gamma\left(a_{1}\right)}{\Gamma\left(a_{1}+b_{1}\right)} \sum_{l=0}^{\infty} \frac{(\kappa ; p, v)_{l}\left(a_{1}\right)_{\sigma l}}{\Gamma(a l+b) \times\left(a_{1}+b_{1}\right)_{\sigma l}} \frac{x^{l}}{l !} \\
= & \frac{\Gamma\left(b_{1}\right) \Gamma\left(a_{1}\right)}{\Gamma\left(a_{1}+b_{1}\right) \Gamma(b)} \sum_{l=0}^{\infty} \frac{(\kappa ; p, v)_{l}\left(a_{1}\right)_{\sigma l} x^{l}}{(b)_{a l}\left(a_{1}+b_{1}\right)_{\sigma l} l !}=\frac{\Gamma\left(b_{1}\right) \Gamma\left(a_{1}\right)}{\Gamma\left(a_{1}+b_{1}\right) \Gamma(b)} \\
& \cdot \sum_{l=0}^{\infty} \frac{(\kappa ; p, v)_{l} \prod_{j=1}^{\sigma}\left(\left(a_{1}+j-1\right) / \sigma\right)_{l} \sigma^{\sigma l}}{\prod_{j=1}^{a}((b+j-1) / a)_{l} a^{a l} \prod_{j=1}^{\sigma}\left(\left(a_{1}+b_{1}+j-1\right) / \sigma\right)_{l} \sigma^{\sigma l}} \frac{x^{l}}{l !} \\
= & \frac{\Gamma\left(b_{1}\right) \Gamma\left(a_{1}\right)}{\Gamma\left(a_{1}+b_{1}\right) \Gamma(b)} \\
& \cdot \sum_{l=0}^{\infty} \frac{(\kappa ; p, v)_{l} \prod_{j=1}^{\sigma}\left(\left(a_{1}+j-1\right) / \sigma\right)_{l}}{\prod_{j=1}^{a}((b+j-1) / a)_{l} l^{a l} \prod_{j=1}^{\sigma}\left(\left(a_{1}+b_{1}+j-1\right) / \sigma\right)_{l}} \frac{x^{l}}{l !} \\
= & \frac{\Gamma\left(b_{1}\right) \Gamma\left(a_{1}\right)}{\Gamma(b) \Gamma\left(a_{1}+b_{1}\right)_{\sigma+1}} \\
& \cdot F_{a+\sigma}\left[(\kappa, p, v), \Delta\left(\sigma ; a_{1}\right) ; \Delta(a ; b), \Delta\left(\sigma ; a_{1}+b_{1}\right) ; \frac{x}{a^{a}}\right] .
\end{aligned}
$$

Corollary 8. By putting $a_{1}=b$ and $\sigma=a \in \mathbb{C}$ in (33) and then using (14), we have

$$
\int_{0}^{1} z_{1}^{b-1}\left(1-z_{1}\right)^{b_{1}-1} \varepsilon_{a, b ; p, v}^{\kappa}\left(x z^{a}\right) d z_{1}=\Gamma\left(b_{1}\right) \varepsilon_{a, b+b_{1} ; p, v}^{\kappa}(x) .
$$

Similarly, we have

$\int_{0}^{1} z_{1}^{a_{1}-1}\left(1-z_{1}\right)^{b-1} \varepsilon_{a, b ; p, v}^{\kappa}\left(x\left(1-z_{1}\right)^{a}\right) d z_{1}=\Gamma\left(a_{1}\right) \varepsilon_{a, b+a_{1} ; p, v}^{\kappa}(x)$.

In general, we get

$$
\begin{aligned}
& \int_{0}^{1}\left(x-z_{1}\right)^{a_{1}-1}\left(z_{1}-t\right)^{b-1} \varepsilon_{a, b ; p, v}^{\kappa}\left(w\left(z_{1}-t\right)^{a}\right) d z_{1} \\
& \quad=\Gamma\left(a_{1}\right)(x-t)^{b+a_{1}-1} \varepsilon_{a, b+a_{1} ; p, v}^{\kappa}\left(w(z-t)^{a}\right) .
\end{aligned}
$$

5.3. Laplace Transform. The well-known Laplace transform [26] of $f\left(z_{1}\right)$ is defined by

$$
\mathbf{L}\left\{f\left(z_{1}\right)\right\}=\int_{0}^{\infty} e^{-s z_{1}} f\left(z_{1}\right) d z_{1} .
$$

Theorem 9. For the function $\varepsilon_{a, b ; p, v}^{\kappa}\left(z_{1}\right)$ in (14), the following Laplace transform holds:

$$
\begin{aligned}
\mathbf{L}\{ & \left.z_{1}^{a_{1}-1} \varepsilon_{a, b ; p, v}^{\kappa}\left(x z_{1}^{\sigma}\right)\right\} \\
= & \frac{\Gamma\left(a_{1}\right)}{\Gamma(b) s^{a_{1}}} F_{\sigma+1} F_{a}\left[(\kappa, p, v), \Delta\left(\sigma ; a_{1}\right) ; \Delta(a ; b) ; \frac{x a^{a}}{(s \sigma)^{\sigma}}\right] \\
& \quad\left(a, \sigma \in \mathbb{N} ; \mathfrak{R}(p)>0, \mathfrak{R}\left(a_{1}\right)>0, \mathfrak{R}(s)>0, \mathfrak{R}(b)>0,\right. \\
& \quad \mathfrak{R}(\kappa)>0, p \geq 0, v \geq 0) .
\end{aligned}
$$


Proof. By using (45) and from (14), we have

$$
\begin{aligned}
\mathbf{L}\left\{z_{1}^{a_{1}-1} \varepsilon_{a, b ; p, v}^{\kappa}\left(x z_{1}^{\sigma}\right)\right\} & =\int_{0}^{\infty} z_{1}^{a_{1}-1} e^{-s z_{1}} \varepsilon_{a, b ; p, v}^{\kappa}\left(x z_{1}^{\sigma}\right) d z_{1} \\
& =\int_{0}^{\infty} z_{1}^{a_{1}-1} e^{-s z_{1}}\left(\sum_{l=0}^{\infty} \frac{(\kappa ; p, v)_{l}}{\Gamma(a l+b)} \frac{x^{l} z_{1}^{\sigma l}}{l !}\right) d z_{1} \\
& =\sum_{l=0}^{\infty} \frac{(\kappa ; p, v)_{l}}{\Gamma(a l+b)} \frac{x^{l}}{l !} \int_{0}^{\infty} z_{1}^{a_{1}+\sigma l-1} e^{-s z_{1}} d z_{1} \\
& =\sum_{l=0}^{\infty} \frac{(\kappa ; p, v)_{l}}{\Gamma(a l+b)} \frac{x^{l}}{l !} \frac{\Gamma\left(a_{1}+\sigma l\right)}{s^{a_{1}+\sigma l}} \\
& =\sum_{l=0}^{\infty} \frac{(\kappa ; p, v)_{l}}{\Gamma(a l+b)} \frac{x}{l !} \frac{\Gamma\left(a_{1}+\sigma l\right)}{s^{a_{1}+\sigma l}} \\
& =\frac{\Gamma\left(a_{1}\right)}{\Gamma(b) s^{a_{1}}} \sum_{l=0}^{\infty} \frac{(\kappa ; p, v)_{l}\left(a_{1}\right)_{\sigma l}}{(b)_{a l}} \frac{\left(x a^{a}\right)^{l}}{\left(s^{\sigma} \sigma^{\sigma}\right)^{l}} \frac{1}{l !} \\
& =\frac{\Gamma\left(a_{1}\right)}{\Gamma(b) s^{a_{1}}} F_{\sigma+1}\left[(\kappa, p, v), \Delta\left(\sigma ; a_{1}\right) ; \Delta(a ; b) ; \frac{x a^{a}}{(s \sigma)^{\sigma}}\right]
\end{aligned}
$$

Corollary 10. By setting $a_{1}=b$ and $\sigma=a$ in (46), we obtain

$$
\int_{0}^{\infty} z^{b-1} e^{-s z} \varepsilon_{a, b ; p, v}^{\kappa}\left(x z^{a}\right) d z=\frac{1}{s^{b}} F_{0}\left[(\kappa, p, v) ;-; \frac{x}{s^{a}}\right] .
$$

5.4. Whittaker Transformation. To determine the Whittaker transforms, we use the following formula:

$\int_{0}^{\infty} t^{\nu-1} e^{-(1 / 2) t} \mathbf{W}_{\eta, \xi}(t) d t=\frac{\Gamma((1 / 2) \pm \xi+v)}{\Gamma(1-\eta+v)}\left(\mathfrak{R}\left(v \pm \xi>-\frac{1}{2}\right)\right.$
Theorem 11. For the function $\varepsilon_{a, b ; p, v}^{\kappa}\left(z_{1}\right)$ in (14), the following Whittaker transform holds:

$$
\begin{aligned}
\int_{0}^{\infty} t^{\rho-1} & e^{-(1 / 2) s t} \mathbf{W}_{\eta, \xi}(s t) \varepsilon_{a, b ; p, v}^{\kappa}\left(\omega z^{\delta}\right) d t \\
= & \frac{\Gamma((1 / 2) \pm \xi+\rho)}{\Gamma(1-\eta+\rho) \Gamma(b) s^{\rho}}{ }_{2 \vartheta+1} \\
& \cdot F_{a+\vartheta}\left[(\kappa, p, v), \Delta\left(\vartheta ; \frac{1}{2} \pm \xi+\rho\right) ; \Delta(a, b), \Delta(\vartheta ; 1-\eta+\rho) ; \frac{\omega a^{a}}{(s \vartheta)^{\vartheta}}\right] \\
& \cdot(a, \vartheta \in \mathbb{N} ; p \geq 0 ; \mathfrak{R}(p)>0, \mathfrak{R}(\rho)>0, \mathfrak{R}(b)>0, \mathfrak{R}(\kappa)>0, v \geq 0) .
\end{aligned}
$$

Proof. By the definition of Whittaker transform, we have from (14)

$$
\begin{aligned}
\int_{0}^{\infty} t^{\rho-1} e^{-(1 / 2) s t} \mathbf{W}_{\eta, \xi}(s t) \varepsilon_{a, b ; p, v}^{\kappa}\left(\omega t^{\vartheta}\right) d t \\
\quad=\int_{0}^{\infty} t^{\rho-1} e^{-(1 / 2) s t} \mathbf{W}_{\eta, \xi}(s t)\left(\sum_{l=0}^{\infty} \frac{(\kappa ; p, v)_{l}}{\Gamma(a l+b)}\right) \frac{\omega^{l} t^{9 n}}{l !} d t \\
=\sum_{l=0}^{\infty} \frac{(\kappa ; p, v)_{l}}{\Gamma(a l+b)} \frac{\omega^{l}}{l !} \int_{0}^{\infty} t^{\rho+\vartheta l-1} e^{-(1 / 2) s t} \mathbf{W}_{\eta, \xi}(s t) d t
\end{aligned}
$$

By putting $v=s t$ and then using the definition of Whittaker transforms, we get

$$
\begin{aligned}
\int_{0}^{\infty} t^{\rho-1} e^{-(1 / 2) t} \mathbf{W}_{\eta, \xi}(s t) \varepsilon_{a, b ; p, v}^{\kappa}\left(\omega z^{9}\right) d t & =\sum_{l=0}^{\infty} \frac{(\kappa ; p, v)_{l} \omega^{l}}{\Gamma(a l+b)} \frac{\Gamma((1 / 2) \pm \xi+\rho+\vartheta l)}{l !}(s)^{-\rho-\vartheta l} \\
& =\frac{1}{s^{\rho}} \sum_{l=0}^{\infty} \frac{(\kappa ; p, v)_{l} \Gamma((1 / 2) \pm \xi+\rho+\vartheta l) \times \Gamma((1 / 2) \pm \xi+\rho) / \Gamma((1 / 2) \pm \xi+\rho)}{\Gamma(a l+b) \times \Gamma(1-\eta+\rho+\vartheta l) \times \Gamma(1-\eta+\rho) / \Gamma(1-\eta+\rho)} \times \frac{1}{l !}\left(\frac{\omega}{s^{\vartheta}}\right)^{l} \\
& =\frac{\Gamma((1 / 2) \pm \xi+\rho)}{\Gamma(1-\eta+\rho) \Gamma(b) s^{\rho}} \sum_{l=0}^{\infty} \frac{(\kappa ; p, v)_{l}((1 / 2) \pm \xi+\rho)_{\vartheta l}}{(b)_{a l}(1-\eta+\rho)_{\vartheta l}} \frac{1}{l !}\left(\frac{\omega}{s^{9}}\right)^{l} \\
& =\frac{\Gamma((1 / 2) \pm \xi+\rho)}{\Gamma(1-\eta+\rho) \Gamma(b) s^{\rho}}{ }_{2 \vartheta+1} F_{a+\vartheta}\left[(\kappa, p, v), \Delta\left(\vartheta ; \frac{1}{2} \pm \xi+\rho\right) ; \Delta(a, b), \Delta(\vartheta ; 1-\eta+\rho) ; \frac{\omega a^{a}}{(s \vartheta)^{9}}\right] .
\end{aligned}
$$

\section{Conclusion}

In our current investigation, we presented an extension of the generalized ML function $\varepsilon_{a, b ; p, v}^{\kappa}\left(z_{1}\right)$ in (14) by utilizing an extension of Pochhammer symbol $(\mu ; \omega, a)_{\eta}$ defined in (9). Further, we have investigated several basic properties of the newly defined function $\varepsilon^{\kappa}{ }_{a, b ; p, v}\left(z_{1}\right)$. The special cases of the main result for $v=0$ can be found in the work of [12]. Thus, the results introduced in this present article are new and an extension of the relating outcomes in the existing literature (see, e.g., [29-31]). The newly defined ML function $\varepsilon_{a, b ; p, v}^{\kappa}\left(z_{1}\right)$ presented in this article will be applicable in different fields of applied sciences. 


\section{Data Availability}

No data was used for this study.

\section{Conflicts of Interest}

The authors declare that they have no competing interests.

\section{Authors' Contributions}

All the authors contributed equally.

\section{Acknowledgments}

The author Thabet Abdeljawad would like to thank Prince Sultan University for funding this work through research group Nonlinear Analysis Methods in Applied Mathematics (NAMAM) group number RG-DES-2017-01-17.

\section{References}

[1] R. P. Agarwal, "A propos d'une note de M. Pierre Humbert," Comptes Rendus de l'Académie des Sciences Paris, vol. 236, pp. 2031-2032, 1953.

[2] P. Humbert, "Quelques resultats relatifs a la fonction de Mittag-Leffler," Comptes Rendus de l'Académie des Sciences Paris, vol. 236, pp. 1467-1468, 1953.

[3] P. Humbert and R. P. Agarwal, "Sur la fonction de MittagLeffler et quelques-unes de ses generalisation," Bulletin of Mathematical Sciences, vol. 77, pp. 180-185, 1953.

[4] G. M. Mittag-Leffler, "Sur la nouvelle fonction $\mathrm{E}_{\alpha}(\mathrm{x})$," Comptes Rendus de l'Académie des Sciences Paris, vol. 137, pp. 554-558, 1903.

[5] G. M. Mittag-Leffler, "Sur la représentation analytique d'une branche uniforme d'une fonction monogène: cinquième note," Acta Math, vol. 29, pp. 101-181, 1905.

[6] A. Wiman, "Uber den Fundamentalsatz in der Teorie der Funktionen $\mathrm{E}^{\alpha}(\mathrm{x})$," Acta Math, vol. 29, no. 1, pp. 191-201, 1905.

[7] A. Wiman, "Über die Nullstellen der Funktionen Ea(x)," Acta Math, vol. 29, pp. 217-234, 1905.

[8] T. R. Prabhakar, "A singular integral equation with a generalized Mittag Leffler function in the kernel," Yokohama Mathematical Journal, vol. 19, pp. 7-15, 1971.

[9] A. K. Shukla and J. C. Prajapati, "On a generalization of Mittag-Leffler function and its properties," Journal of Mathematical Analysis and Applications, vol. 336, no. 2, pp. 797-811, 2007.

[10] G. Rahman, D. Baleanu, M. A. Qurashi, S. D. Purohit, S. Mubeen, and M. ARshad, "The extended Mittag-Leffler function via fractional calculus," The Journal of Nonlinear Sciences and Applications, vol. 10, no. 8, pp. 4244-4253, 2017.

[11] M. A. Chaudhry and S. M. Zubair, On a Class of Incomplete Gamma Functions with Applications, Chapman and Hall (CRC Press Company), Boca Raton, London, New York and Washington, D. C, 2001.

[12] J. Choi, R. K. Parmar, and P. Chopra, "Extended MittagLeffler function and associated fractional calculus operators," Georgian Mathematical Journal, vol. 2019, pp. 1-11, 2019.

[13] G. Rahman, A. Ghaffar, S. Mubeen, M. Arshad, and S. U. Khan, "The composition of extended Mittag-Leffler functions with pathway integral operator," Advances in Difference Equations, vol. 2017, no. 1, p. 176, 2017.

[14] G. Rahman, P. Agarwal, S. Mubeen, and M. Arshad, "Fractional integral operators involving extended Mittag-Leffler function as its Kernel," Boletín de la Sociedad Matemática Mexicana, vol. 24, no. 2, pp. 381-392, 2018.

[15] S. Araci, G. Rahman, A. Ghaffar, and K. S. N. Azeema, "Fractional calculus of extended Mittag-Leffler function and its applications to statistical distribution," Mathematics, vol. 7, p. $248,2019$.

[16] G. Rahman, K. S. Nisar, J. Choi, S. Mubeen, and M. Arshad, "Pathway fractional integral formulas involving extended Mittag-Leffler functions in the kernel," Kyungpook National University, vol. 59, pp. 125-134, 2019.

[17] H. M. Srivastava, G. Rahman, and K. S. Nisar, "Some extensions of the pochhammer symbol and the associated hypergeometric functions," Iranian Journal of Science and Technology, Transaction A: Science, vol. 43, no. 5, pp. 2601-2606, 2019.

[18] H. M. Srivastava, A. Cetinkaya, and O. I. Kiymaz, “A certain generalized Pochhammer symbol and its applications to hypergeometric functions," Applied Mathematics and Computation, vol. 226, pp. 484-491, 2014.

[19] M. Arshad, S. Mubeen, K. S. Nisar, and G. Rahman, "Extended Wright-Bessel function and its properties," Korean Mathematical Society, vol. 33, no. 1, pp. 143-155, 2018.

[20] M. Arshad, J. Choi, S. Mubeen, K. S. Nisar, and G. Rahman, "A new extension of the Mittag-Leffler function," Communications of the Korean Mathematical Society, vol. 33, no. 2, article 549560, 2018.

[21] S. Mubeen, G. Gahman, K. S. Nisar, J. Choi, and M. Arshad, "An extended beta function and its properties," Far East Journal of Mathematical Sciences, vol. 102, no. 7, pp. 1545-1557, 2017.

[22] H. M. Srivastava, A. Tassaddiq, G. Rahman, K. S. Nisar, and I. Khan, "A new extension of the $\tau$-gauss hypergeometric function and its associated properties," Mathematics, vol. 7, no. 10, p. 996, 2019.

[23] K. S. Nisar, G. Rahman, and Z. Tomovski, "On a certain extension of Riemann-Liouville fractional derivative operator," Korean Mathematical Society, vol. 34, no. 2, pp. 507522, 2019.

[24] M. Bohner, G. Rahman, S. Mubeen, and K. S. Nisar, "A further extension of the extended Riemann-Liouville fractional derivative operator," Turkish Journal of Mathematics, vol. 42, no. 5, pp. 2631-2642, 2018.

[25] G. Rahman, S. Mubeen, K. S. Nisar, and J. Choi, "Certain extended special functions and fractional integral and derivative operators via an extended beta functions," Nonlinear Functional Analysis and Applications, vol. 24, no. 1, pp. 1-13, 2019.

[26] I. N. Sneddon, The Use of the Integral Transforms, Tata McGraw-Hill, New Delhi, 1979.

[27] E. M. Wright, "The asymptotic expansion of the generalized hypergeometric function," Journal of the London Mathematical Society, vol. 10, pp. 286-293, 1935.

[28] E. M. Wright, "The asymptotic expansion of the generalized hypergeometric function," Journal of the London Mathematical Society, vol. 46, pp. 389-408, 1940.

[29] R. Gorenflo, A. A. Kilbas, F. Mainardi, and S. V. Rogosin, Mittag-Leffler Functions, Related Topics and Applications, Springer Monograph Math, Springer, Heidelberg, 2014. 
[30] H. J. Haubold, A. M. Mathai, and R. K. Saxena, "Mittag-Leffler functions and their applications," Journal of Applied Mathematics, vol. 2011, Article ID 298628, 51 pages, 2011.

[31] A. A. Kilbas, M. Saigo, and R. K. Saxena, "Generalized MittagLeffler function and generalized fractional calculus operators," Integral Transforms and Special Functions, vol. 15, no. 1, pp. 31-49, 2004. 